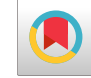

\title{
Association of cagA, cagC, virB2, and vacA Subtypes of Helicobacter pylori with Adenocarcinoma Development in Iranian Patients
}

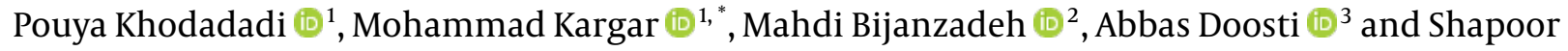 \\ Aghaei (iD) 4 \\ ${ }^{1}$ Department of Microbiology, Jahrom Branch, Islamic Azad University, Jahrom, Iran \\ ${ }^{2}$ Department of Medical Genetic, Ahvaz Jundishapur University of Medical Sciences, Ahvaz, \\ ${ }^{3}$ Biotechnology Research Center, Shahrekord Branch, Islamic Azad University, Shahrekord, Iran \\ ${ }^{4}$ Department of Internal Medicine, Yasuj University of Medical Sciences, Yasuj, Iran \\ "Corresponding author: Department of Microbiology, Jahrom Branch, Islamic Azad University, Jahrom, Iran. Tel: +98-9173149203, Email: microkargar@gmail.com \\ Received 2020 January 25; Revised 2020 July 09; Accepted 2020 July 24.
}

\begin{abstract}
Background: Gastric cancer has been introduced as the second cause of cancer death worldwide. Helicobacter pylori infection is considered one of the main risk factors for this type of cancer, so that it has been classified as group I carcinogens.

Objectives: The present research intended to examine the prevalence of $\operatorname{cagA}, \operatorname{cagC}, \operatorname{virB2}$, vacA, and genotype distribution in $H$. pylori-infected biopsies and adenocarcinoma cases.

Methods: Thirty-four H. pylori gastric biopsies taken from Western Iranian patients that were diagnosed as gastritis, gastric ulcers, and adenocarcinoma were used in this study. Two samples were taken from each patient. These samples were selected based on endoscopic observations and histological examinations. The presence of $H$. pylori was confirmed by the Rapid Urease test (RUT) and the ure C gene by the polymerase chain reaction (PCR) technique. Then, specific primers for vacA and cagPAI were used for genotyping H. pylori by PCR-typing.

Results: The obtained results showed that $86.8 \%$ of the samples were $H$. pylori-positive. Moreover, the cagA gene prevalence was $51.50 \%$ in the samples. In addition, the adenocarcinoma outcome was significantly related to all selected genes. Likewise, some gastric diseases such as gastric ulcers, duodenal ulcer (DU), gastritis, lymphoid, and gastroesophageal reflux disease (GERD) were observed in adenocarcinoma cases. It was also found that the vacAs1mii1 genotype plays an important role in gastric malignancies. The most frequent vacA genotype in the H. pylori-infected isolates was s1mi11, and the observed frequency of vacA and cagA genes in adenocarcinoma was statistically significant.

Conclusions: The findings showed that $H$. pylori vacA and cagA gene virulence factors are associated with adenocarcinoma in Western Iranian patients.
\end{abstract}

Keywords: Gastric Adenocarcinoma, cagPAI, Helicobacter pylori, vacA Genotypes, s1m1i1

\section{Background}

Helicobacter pylori has been introduced as one of the microaerophilic spiral-shaped Gram-negative bacteria, which belongs to Epsilonoprotobacteria, colonizing in the gastric mucosa layer of $50 \%$ of the population during their lifetime worldwide (1-3). Its transmission mainly occurs through the fecal-oral and oral-oral routes or contaminated sources such as contaminated water supply; however, the observed mechanism varies from person to person (2). Studies have shown a correlation between the infection caused by this bacterium and different kinds of gastric conditions like chronic superficial gastritis, gastric inflammation, chronic active gastritis, duodenal ulcers, gastric cancer, and peptic ulcer disease (4).
Some clinical symptoms in patients with gastroduodenal disease are vomiting, gastrointestinal bleeding, and weight loss. Moreover, some people suffer from stomach ulcers, dysphagia, difficulty swallowing, reflux, atrophy, and indigestion (5). In addition, the worldwide mortality rate due to gastric cancer remains high, specifically in Asia and some developing countries (2). Although gastric cancer occurrence is slowly decreasing in the West, it remains the second most common cause of death from cancer all over the world. It has been estimated that gastric cancer is responsible for 700,000 cases of $H$. pylori-associated morbidity around the world (6). Despite the increased pervasiveness of $H$. pylori worldwide, the infected people are mostly asymptomatic. Therefore, it is evident that the risk 
of developing gastric carcinoma is largely higher in H. pylori-infected people than in uninfected ones (7). For this reason, the treatment of infected individuals has become a big challenge in medical services (2).

In general, patients and asymptomatic individuals acquire H. pylori during childhood or adolescence (8). However, statistics indicate that the prevalence of this bacterium is higher in developing countries than in developed countries (90\% versus 25\%) (9). Based on previous studies, the prevalence of infection in Iran ranges from 60 to $90 \%(2,10)$. The risk of human infections with $H$. pylori depends on several factors, including age, gender, diet, type of bacterium, environmental factors, genetic diversity of bacterium, and host factors $(11,12)$. In addition, $H$. pylori has the greatest heterogeneity among enteric bacteria in terms of genetic content so that conserved gene sequences have diversities in many strains. Hence, H. pylori infection depends on different factors such as environmental conditions, hygiene level of society, and the characteristics of the organism that affect disease outcomes, including viral genes like vacA, iceA, dupA, cagA, babA, and homB.

Many virulence factors of $H$. pylori, such as cytotoxinassociated gene A ( $\operatorname{cag} A)$, vacuolating cytotoxin A (vacA), urease, adhesins, and flagella, are involved in its pathogenesis. Indeed, $H$. pylori adhesins contain outer membrane proteins (OMPs), including DupA, IceA, OipA, AlpA/B, HomB, HopZ, OipA, SabA, and BabA $(11,13,14)$ that make this bacterium as one the most prevalent pathogens in human stomach diseases $(15,16)$. Some of these genes seem to be linked with gastric inflammation. According to research, cag pathogenicity island (cagPAI), cagA, and vacA have an essential role in the stimulation of epithelial cells and Stimulates innate immune system (13). Besides, cagA is a major $H$. pylori virulence factor in cagPAI and one of the most significant markers for the presence of cagPAI in H.pylori strains. Some studies indicated special clinical features in people with gastroduodenal disorders by infection with cagA-positive H. pylori strains.

Clinical symptoms such as vomiting, difficulty swallowing, and abdominal pain make it possible to detect bacterial infection in the early stage. On the other hand, clinical manifestations are a useful way to assess bacterial surveillance. However, because of increased $H$. pylori antibiotic resistance, bacteria diagnosis in the early stages may be the best way to eradicate the disease. Moreover, estimates show that cagPAI comprises about 40 genes, among which seven genes comprise the type IV secretion system (T4SS). Therefore, T4SS transfers CagA into the host epithelial cells and induces cell toxicity and vacuolation (17). CagA is also a surface protein with high immunization potential, which can result in the deformation of spindleshaped cells after entrance into stomach epithelial cells.
Entering the CagA protein into target cells and cell signaling interference can increase the pathogenicity and express cagPAI-related genes. In addition, cagA expression in the strains can lead to several morphologic changes, including bacterial generation and apoptosis in host cells. It was found that cagA-positive strains have high potency to nesting, erosion, and inflammation (18). Furthermore, vir genes are part of cagPAI and have a similar structure to T4SS called cagPAI and ComB, which are located before and after $d u p A$ locus, and thus have been considered as third T4SS (19).

The vacA gene includes three main regions of the signal zone that are characterized by $s 1$ and $s 2$, the middle region ( $m 1$ and $m 2$ ), and the intermediate region (i1, $i 2$, and i3). These polymorphic zones exhibit different combinations, some of which have the highest activity to make human gastric diseases $(20,21)$. Due to mosaicism, a combination of different distinct regions of $v a c A s / m / i$ resulted in eight possible combinations (subtypes) (11). Because of vacA mosaicism, different $H$. pylori strains may show different pathogenicity capacities (22). In most studies conducted in Iran, the $i$ region vacA genotyping has received less attention. In this research, we considered all three $v a c A$ gene regions. Our results interestingly implicated that $m 1$ and $i 1$ alleles had a significant relationship with gastritis and adenocarcinoma. However, $i 1$ allele has more activity than $i 2$. One of the studies in the field showed the relationship of a combination of strains, i1 allele, and s1m1 allele with more cytotoxic activity and peptic ulceration. The sii1 genotype induces the severe vacuolating activity of vacA and thus can lead to metaplasia and inflammation compared to $\$ 1 i 2$ or $s 2 i 2(23)$.

\section{Objectives}

The present study aimed to conduct a survey on H. pylori genotyping and examine survey relationship between different $H$. pylori genotypes and different vacA alleles in gastric disease incidence.

\section{Methods}

\subsection{Collection of Patients' Samples}

Gastric biopsy specimens were collected under the decision of gastroenterologists following the upper endoscopy of 68 patients with gastroduodenal symptoms during six years. Thirty-four specimens were diagnosed as adenocarcinoma and the remaining samples were obtained from patients with gastric ulcers and gastritis. A questionnaire, including demographic data and medical history, was completed for each patient. Then, all of 
the samples were tested by the Rapid Urease test (RUT) (Baharafshan, Iran) and consequently were subjected to pathological examinations. The biopsy samples were kept at $-20^{\circ} \mathrm{C}$ to be used later. All subjects of the study signed informed consent forms before endoscopy. In the next stage, a disinfected endoscope was used to take two biopsy specimens from the antrum. Giemsa reagent was utilized to stain one piece of each specimen to screen for H. pylori. All samples were placed in $0.1 \mathrm{~mL}$ of sterile saline solution and transferred to the Genetic Laboratory of Ahvaz Jundishapur University of Medical Sciences for additional analysis.

\subsection{Extraction of Genomic DNA From Helicobacter pylori}

The DNA extraction kit manufactured by Qiagen DNA Mini Kit, Iran, was used to isolate the DNA from biopsy samples following the manufacturer's instructions. Then, the quantity and quality of the resulting extracted DNA were examined on $2 \%$ agarose gel electrophoresis and NanoDrop (Nano-drop Technologies, Wilmington, DE, USA) at the wavelength of $260 / 280 \mathrm{~nm}$. The extracted DNA was utilized for subsequent experiments of the polymerase chain reaction (PCR). Then, ureC was primarily amplified by using specific primers for the detection of H. pylori DNA in all samples. The sequence of the $\operatorname{cag} A, \operatorname{cagC}$, virB2, and vacA genes was deposited in the GenBank database. Finally, Gene Runner 3.5 was employed to design specific forward and reverse primers to the amplified genes, as given in Table 1.

\subsection{Amplification of vacA ( $s 1, s 2, m 1, m 2, i 1, i 2)$ and cagA Genes}

The PCR was performed using a thermal cycler (Mastercycler Gradient, Eppendorf, Germany) in a total reaction volume of $25 \mu \mathrm{L}$ consisting of $2.5 \mu \mathrm{L}$ of $2 \mathrm{mM} \mathrm{MgCl}_{2}, 2.5 \mu \mathrm{L}$ of 10x PCR buffer, and $100 \mathrm{mM}$ dNTPs of each forward and reverse primer, one unit of Taq DNA polymerase, and $1 \mu \mathrm{g}$ of DNA sample (all chemicals from Fermentas, Thermo Fisher Scientific, Germany). Then, H. pylori strain ATCC 43504 was exploited as a positive control. A cagA negative strain purchase from Ahvaz Jundishapur University of Medical Sciences. It should be noted that PCR temperature conditions involved an initial denaturation at $95^{\circ} \mathrm{C}$ for $5 \mathrm{~min}$, followed by 35 cycles, denaturation at $94^{\circ} \mathrm{C}$ for $1 \mathrm{~min}$, annealing at $61^{\circ} \mathrm{C}$ for $1 \mathrm{~min}$, and extension at $72^{\circ} \mathrm{C}$ for $1 \mathrm{~min}$. Then, we programmed the resulting extension phase at $72^{\circ} \mathrm{C}$ for $5 \mathrm{~min}$, and eventually, the amplified samples were held at $15^{\circ} \mathrm{C}$.

\subsection{Statistical Analysis}

Statistical analysis was done using SPSS 20 software (SPSS Inc., Chicago, IL, USA). The chi-square test and Fisher's exact two-tailed tests were used to assess any significant relationship between the prevalence of $H$. pylori strains and

\begin{tabular}{|c|c|c|}
\hline Gene & Primer Sequence $5^{\prime} \rightarrow 3^{\prime}$ & $\begin{array}{l}\text { PCR Amplicon } \\
\text { Size, bp }\end{array}$ \\
\hline \multirow{2}{*}{ ureC } & F: AAG CTT TTA GGG GTG TTA GGGGTTT & \multirow{2}{*}{296} \\
\hline & R: AAG CTT ACT TTC TAA CAC TAA CGC & \\
\hline \multirow{2}{*}{$\operatorname{cag} A$} & F: ATA ACA GGC AAG CTT TTG AGG & \multirow{2}{*}{349} \\
\hline & R: TGC AAA AGA TTG TTT GGC AGA & \\
\hline \multirow{2}{*}{ vacAs1 } & F: CTC TCG CTT TAG TAG GAG C & \multirow{2}{*}{213} \\
\hline & R: CTG CTT GAA TGC GCC AAA C & \\
\hline \multirow{2}{*}{ vacAs2 } & F: GCT AAC ACG CCA AAT GAT CC & \multirow{2}{*}{199} \\
\hline & R: CTG CTT GAA TGC GCC AAA C & \\
\hline \multirow{2}{*}{ vacAm1 } & F: GGT CAA AAT GCG GTC ATG G & \multirow{2}{*}{290} \\
\hline & R: CCA TTG GTA CCT GTA GAA AC & \\
\hline \multirow{2}{*}{ vacAm2 } & F: GGA GCC CCA GGA AAC ATT G & \multirow{2}{*}{352} \\
\hline & R: CAT AAC TAG CGC CTT GCA C & \\
\hline \multirow{2}{*}{ vacAi1 } & F: GTT GGG ATT GGG GGA ATG CCG & \multirow{2}{*}{426} \\
\hline & R: TTA ATT TAA CGC TGT TTG AAG & \\
\hline \multirow{2}{*}{ vacAi2 } & F: GTT GGG ATT GGG GGA ATG CCG & \multirow{2}{*}{432} \\
\hline & R: GAT CAA CGC TCT GAT TTG A & \\
\hline \multirow{2}{*}{$\operatorname{cagC}$} & F: ATGAAGTTTTTTACAAGAATC & \multirow{2}{*}{360} \\
\hline & R: TTAGCTTGCTCCTCCACTCTC & \\
\hline \multirow{2}{*}{ virB2 } & F: AAGATGAAAACAAAACATAAAG & \multirow{2}{*}{318} \\
\hline & R: CATCTAAAATAACCATTGAC & \\
\hline
\end{tabular}

their genotypes. A P value of $<0.05$ was considered statistically significant.

\section{Results}

The DNA samples derived from gastric biopsy were $H$. pylori-positive, according to the RUT and Giemsa staining. All of the isolates were confirmed by the ureC gene PCR assay, as well. The cagA, cagC, virB2, and vacA PCR products were analyzed on $2 \%$ agarose gel electrophoresis (Figure 1 ). Moreover, $20.60 \%$ of women were H. pylori-positive, while $47.10 \%$ of men were infected $(P=0.221)$. However, no association was found between the use of tobacco and alcohol and $H$. pylori infection. Additionally, this infection was higher in patients aged 50 years or lower (39.70\%) than in older ones (27.90\%), although the relationship was not significant $(\mathrm{P}=0.338)$. Figure 2 presents a summary of the co-occurrence of gastric diseases, including lymphoid, metaplasia, duodenal ulcer (DU), gastritis, non-ulcer disease (NUD), gastric ulcer(GU), and gastroesophageal reflux disease (GERD) with adenocarcinoma.

Using the PCR-electrophoresis analysis, the presence of the cagPAI region (with cagA, $\operatorname{cagC}$, and virB2) was de- 


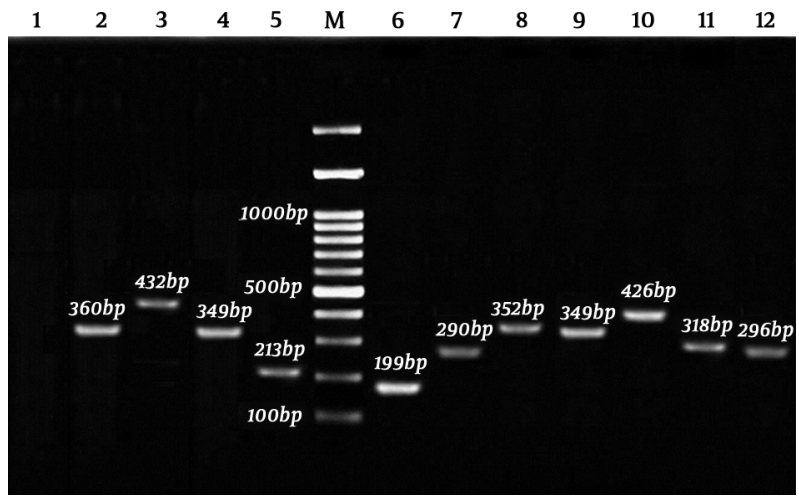

Figure 1. Amplification of $\operatorname{cag} A, \operatorname{cag} C$, virB2, and $v a c A$ alleles and subtypes using PCRelectrophorestic identification of amplified products of Helicobacter pylori: 1, negative control; 2, cagC 360 bp; 3, vacAi2 432 bp; 4, cagA 349 bp; 5, vacAs1 213 bp; 6, vacAs2 $199 \mathrm{bp} ;$ 7, vacAm1 $290 \mathrm{bp} ; 8$, vacAm2 $352 \mathrm{bp} ;$ 9, cagA positive control $349 \mathrm{bp} ; 10$, vacAi1; 426 bp; 11, virB2 318 bp; 12, ureC 296 bp; M, 100 bp marker.

termined in all collected samples, and half of them were adenocarcinoma-positive. Figure 3 depicts the presence of $\operatorname{cag} A, \operatorname{cag} C$, and virB2 in adenocarcinoma and gastritis. The obtained data showed that the presence of $\operatorname{cag} A(\mathrm{P}=$ 0.0.002), cagC (0.002), and virB2 (0.001) genes had a significant association with adenocarcinoma outcome. In gastritis, the detection of $\operatorname{cagA}$, $\operatorname{cag} C$, and virB2 genes was calculated as $25.00 \%(\mathrm{P}=0.009), 22.10 \%(\mathrm{P}=0.019)$, and $16.20 \%(P=0.013)$, respectively. Notably, $16.50 \%$ of the $51.50 \%$ cagA-positive samples were from females and $35.30 \%$ from males. However, no significant relationship was found between the cagA gene and gender $(\mathrm{P}=0.249)$

Observations indicated that $86.8 \%$ of 68 collected samples were detected as vacA-positive $(\mathrm{P}=0.001)$. It was found that s1m1i1 (22.1\%) and s2m2i2 (13 cases) were the most observed genotypes among the samples. However, in the adenocarcinoma samples, the most observed genotypes were s1m1i1 (22.60\%; P = 0.008) and s2m1i1 (8.80\%; P = 0.012), which were associated with adenocarcinoma occurrence. Moreover, $m 1$ and $i 1$ alleles were significantly contributed to adenocarcinoma and gastritis, but no association was observed between $m 1$ and $i 1$ alleles and duodenum ulcer (Figure 4). In addition, statistical analysis showed a significant difference between the s1m1i1 genotype and adenocarcinoma patients (Figure 5).

\section{Discussion}

As known, $H$. pylori is a worldwide infection with variable prevalence in different geographic regions. In 1994, the International Agency for Research on Cancer (IARC) introduced H. pylori as one of the class I carcinogens and dan- gerous factors for stomach cancer $(16,24)$. It was considered an etiologic agent of gastritis and the main factor in $5.5 \%$ of common gastric cancers and lymphoid malignancies (25). According to research, 75\% of gastric cancer cases do not occur in the absence of H. pylori. Based on Iranian research, almost $90 \%$ of adults and more than $50 \%$ of children under the age of 15 are infected with $H$.pylori (26). The identification of individuals at high risk of gastric cancer that may enter a surveillance and intervention program during the precancerous process is the most suitable strategy for decreasing mortality due to this malignancy.

This study showed an association between different genotypes of vacA genes in $H$. pylori-positive Iranian samples who were suffering from adenocarcinoma and gastric diseases. Therefore, it was focused on the presence of the main virulence genes like vacA allele and cagPAI (cagA, $\operatorname{cag} C$, virB2) and also their correlation with gastrointestinal diseases. The vacA gene-encoded protoxin with $140 \mathrm{KD}$, which converted into a mature toxin with $88 \mathrm{KD}$ after secretion. The vacA gene polymorphisms caused cell toxicity resistance. Diversity in the $s, m$, and $i$ regions of the vacA gene influenced the VacA protein. Specifically, a variety of $s$ and $i$ subunits promoted effective vacuolation, whereas the $m$ region contributed importantly to the vacuolated cell diagnosis via an effective connection of the toxin to the host cell $(25,27,28)$. In addition to genotyping, the present study surveyed the relevance of adenocarcinoma outcome with another gastric disease. Therefore, it was observed that adenocarcinoma had a significant correlation with lymphoid, duodenal ulcer, gastritis, and MASS, but there was no significant correlation between metaplasia, NUD, and GERD.

These data showed that persons who are suffering from gastric ulcer, duodenal ulcer, gastritis, and lymphoid but had not been untreated were at the increased risk of gastric cancer. Similar to the obtained data by Farshad et al. (29), we found that $51.5 \%$ of the collected specimens with gastric infection are positive for vacA genes. Moreover, a correlation was observed between vacA genotypes and some gastric diseases such as adenocarcinoma, NUD, and gastritis. In addition, Souod et al. (30) reported the same result as achieved in the current data. However, some previous studies showed that vacA genotypes are not useful markers to anticipate clinical outcomes. As an example, Jafari et al. did not find any association between vacA genotypes and gastric disease (11). Moreover, the prevalence of $\operatorname{cagA}$ positive strains in the southwest of Iran was $95.03 \%$, so that the authors reported that East Asian strains were dominant in this region. The results of several studies suggested that the prevalence of bacterial types could also change over a year (3).

The comparison of cagA in East Asian countries and 


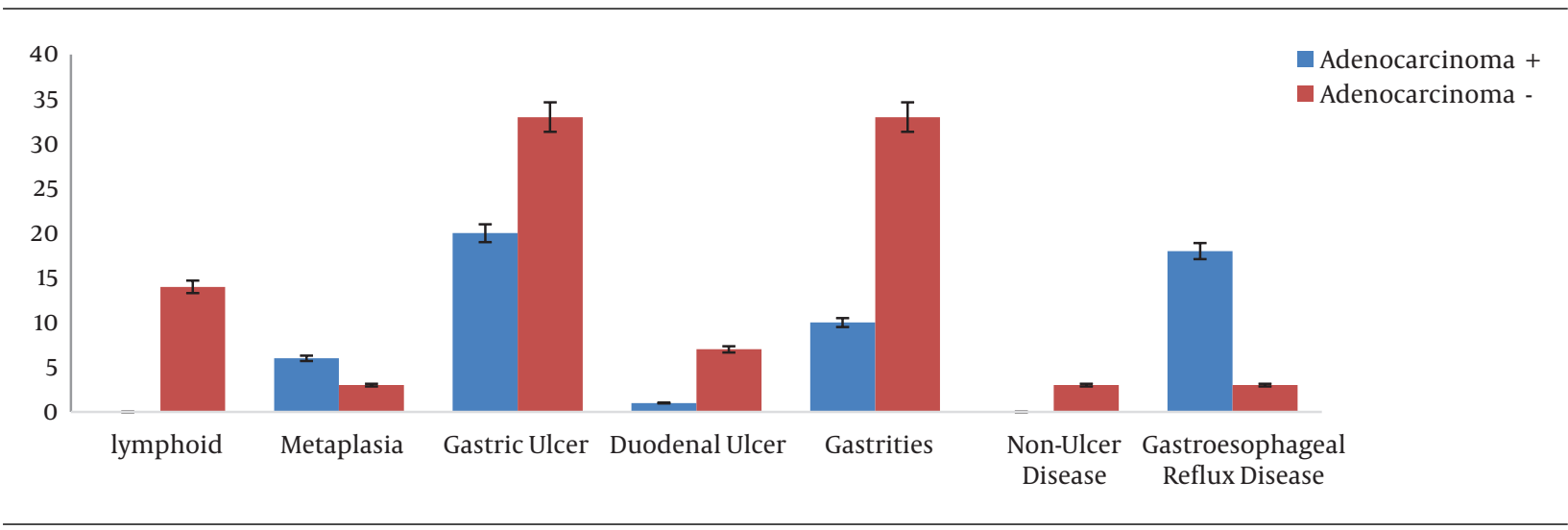

Figure 2. Correlation between adenocarcinoma and gastric disease outcomes

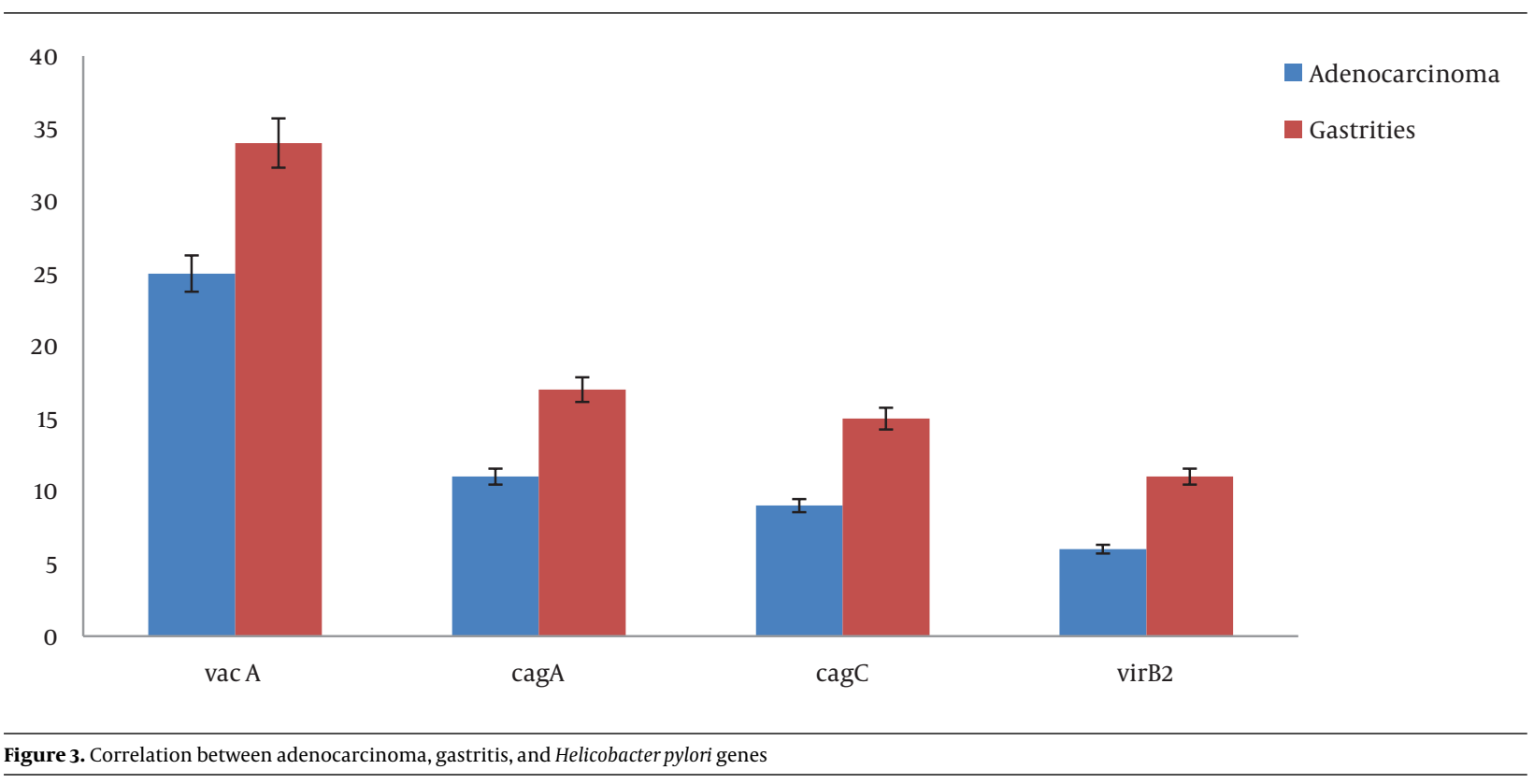

western countries showed that gastric cancer is more strongly associated with $H$. pylori strains carrying East Asian cagA in geographical regions where two different strains coexist (25). In this regard, the frequency of the cagA gene in Malaysia was reported to be higher than $94 \%$ (30). In addition, cagA prevalence in China and neighboring countries such as India was reported to be $93.2 \%$ and $96.2 \%$, respectively (12). Such a variety in the cagA gene frequency in different countries is due to differences in the infected population, geographic condition, and strains' genetic diversity. Kamogawa-Schifter et al.'s study (31) indicated that the prevalence of the cagA gene in people suffering from duodenal ulcers and gastric cancer was 78\% and $86 \%$, respectively. Similarly, the $65.50 \%$ frequency of cagA was found in peptic ulcer isolates in a study carried out in
Turkey (32). Furthermore, cagA pervasiveness in Mexican patients was reported to be $57 \%$ in chronic gastritis, $58.3 \%$ in gastric cancer, and $61.4 \%$ in gastric ulcer (33).

In the present research, cagA-positive strains isolated from patients suffering from peptic ulcers, peptic cancer, and gastritis were $68.60 \%, 68.60 \%$, and $48.6 \%$, respectively. The frequency of cagPAI genes, vacA in peptic ulcer, and adenocarcinoma equaled $35.5 \%$ that is more than in gastritis and there were significant differences in the presence of these genes between adenocarcinoma and gastritis. Such different percentages between the mentioned genes and their genotypes, as well as differences among various countries, may be related to the geographic spread. Therefore, it may be concluded that the co-presence of cagA and vacA may worsen the disease outcome and increase 


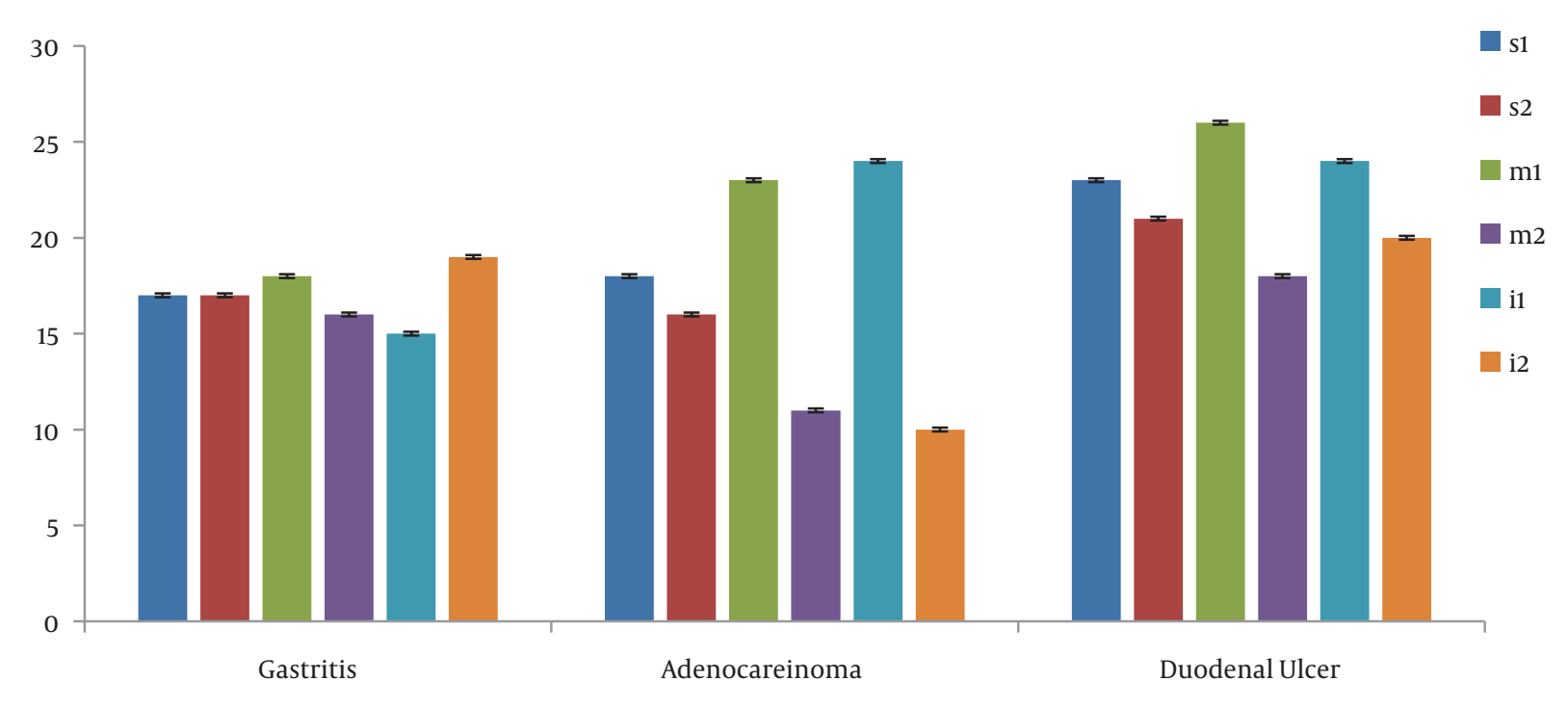

Figure 4. The association between vacA alleles and some gastric diseases

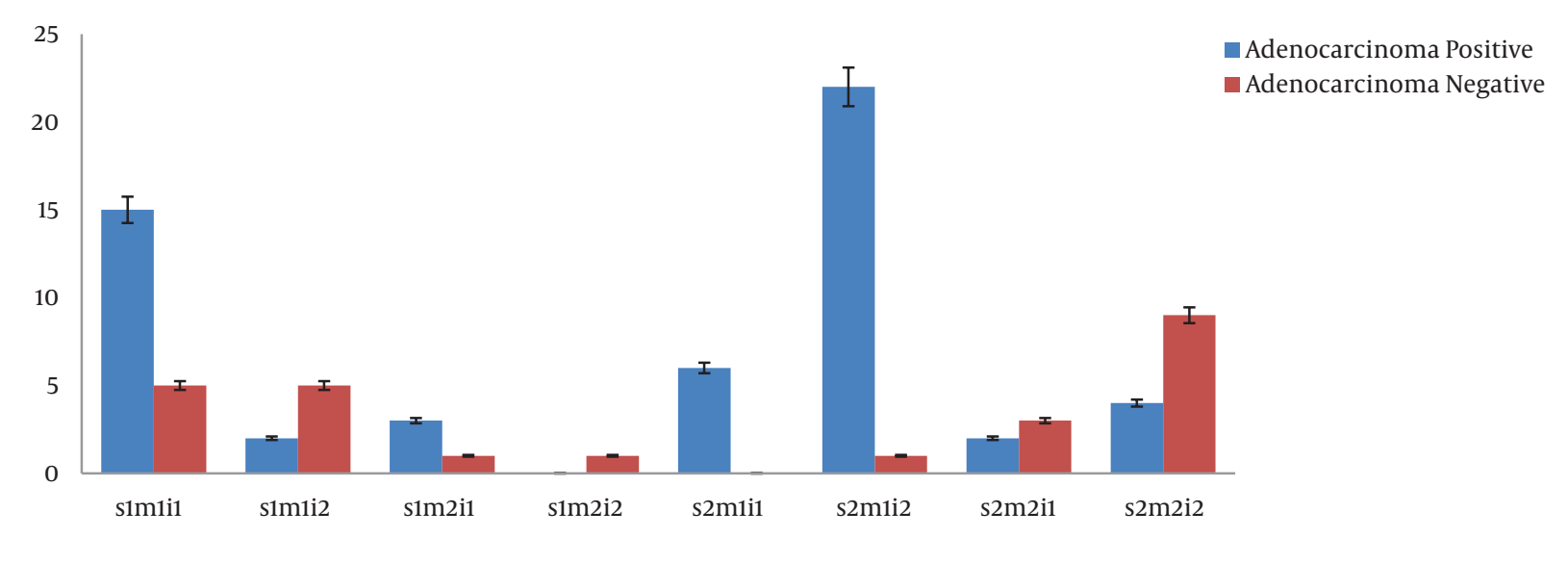

Figure 5. The association between vacA genotypes and adenocarcinoma outcome

the pathogenesis $(25,27)$. Hence, this study considered the prevalence of cagPAI in adenocarcinoma and gastritis and found that most cagA-positive people were also vacApositive; however, all cagA-negative people were not necessarily vacA-negative. Despite the difference in gene frequency, the existence of cagPAI and vacA could be considered a marker for disease intensity, and thus the importance of other factors such as environmental factors could not be ignored (32).

The association between these alleles, disease intensity and type of diseases such as gastritis, gastric ulcer, and adenocarcinoma was investigated, as well. It was found that the frequency of vacA alleles was almost equal in adenocarcinoma, gastritis, peptic ulcer, lymphoid, DU, NUD, metaplasia, and presence of masses, and no significant difference was observed (Figure 4). However, vacAi1 and va$c A m 1 / m 2$ had a significant correlation with gastrointestinal diseases in some cases. In this context, vacAi1 and $m 1$ alleles were significantly related to adenocarcinoma. Moreover, there was a significant relationship between vacAm2 and vacAi1 alleles and gastritis in patients suffering from gastritis (34).

Based on various research on the equality of $s 1 / s 2$ alleles, it is of high importance to survey other regions of the vacA gene. According to the current study, among va$c A m$ alleles, $m 1$ had a higher frequency than $m 2$. Moreover, $m 1$ with higher frequency than $m 2$ was found in adenocarcinoma and gastritis cases, which was statistically sig- 
nificant. These results are consistent with the investigations conducted in Japan, China, and South Korea, where the high frequency of $m 1$ allele was reported in adenocarcinoma and gastritis (34), although it did not match the observed results in Vietnam and Hong Kong(19). It seems that the $m 1$ allele has a high potential compared to $m 2$, although it is not sufficient to diagnose high-risk patients. Moreover, a high frequency of the $i 1$ allele was observed in adenocarcinoma cases in comparison with i2, and this difference was significant. Our data are similar to those obtained in Chinese adenocarcinoma patients (35).

The results of this research and other studies carried out in various parts of the world showed that the $v a$ cAi1 and vacAm1 alleles had a positive impact on bacterial pathogenicity. These alleles have a significant correlation with gastritis and adenocarcinoma (30). According to the results from $46 \mathrm{H}$. pylori-positive samples (67.60\%), 37 cases carried the vacA gene, and $29.40 \%$ of them had the vacAs1mii1 genotype. This genotype was considered to be dominant. It was also reported that the $H$. pylori s1m1i1 genotype had a higher potential for vacuolation than other genotypes such as s2m2i2 and s1m2i2. Most of the isolated strains in this research had s1m1i1-dominant genotypes, similar to studies performed in Afghanistan and Iran (36). On the other hand, it is inconsistent with the study conducted in Nigeria. In Nigerian patients, the s1m1i1 genotype accounted for high pathogenicity with the lowest frequency that was against the high pervasiveness of $H$. pylori in this population. However, there was no association with some diseases such as duodenal ulcers and gastric lymphoma (37). Among the reviewed genotypes, $H$. pylori $\operatorname{cag} A^{+}$, s1m1i1 strains had a high frequency in patients with adenocarcinoma. Furthermore, $c a g A^{-}$and $s 2 m 2 i 2$ had a significantly negative correlation with adenocarcinoma outcome.

Among eight examined genotypes of $\mathrm{cagA}^{+}$, s1mii1 (44.10\%) and s2m1i1 (17.60\%) had the highest frequency in patients with adenocarcinoma. The above-mentioned genotypes had a significant correlation with adenocarcinoma. Our data are consistent with a study carried out by Amer et al. in Egypt (38). Even though the results of some investigations conducted in other parts of the world are different from ours (37), the most common vacA genotypes were $s 1 m 1$ and $s 2 m 2$ in Cuban patients. In brief, in H. pylori infections, some pathogenic factors such as environmental factors, genetic elements of the host cell, and bacterial pathogenicity can affect the disease outcome. It seems that various $H$. pylori strains can cause diverse forms of gastroduodenal disease. It should be noted that the current study used $c a g A, c a g c, v a c A$, and virB2 genotypes to identify $H$. pylori strains isolated from patients. The vacApositive strains isolated from ulcer patients were signifi- cantly more than those from non-ulcer patients. However, any significant differences were not found in the frequency of positive cagA isolates between ulcer patients and nonulcer patients.

Therefore, based on our results and others from various countries such as Egypt, vacAi1, and $m 1$ play important roles in gastrointestinal malignancies. Hence, the intermediate regions of the vacA gene can be used as an indicator used to predict $H$. pylori diseases, including gastric cancer (38). Additionally, as the geographical region can affect the prevalence of vacA genotypes, it is highly proposed to clarify the population-specific distribution of hotspots genotypes by other specialists. Furthermore, more investigations in various parts of the world are proposed to illustrate the pathogens such as H. pylori involved in gastroduodenal diseases.

\subsection{Conclusions}

The present study addressed the prevalence of $H$. pylori virulence factors and adenocarcinoma cases in people with gastric cancer for the first time in Iran. The results showed that none of the treatments of stomach-associated diseases could lead to adenocarcinoma and gastric cancer. Therefore, it is proposed to treat bacterial infections around the world and prevent worldwide death caused by cancer. For this reason, designing an effective vaccine to prevent gastric cancer, especially in people susceptible to the disease, seems to be necessary to decrease the cost of treatment. Although in most studies, the $i$ region vacA genotyping has received less attention, the current results interestingly showed that the $i 1$ allele had a significant relationship with gastritis and adenocarcinoma. The identification of individuals at high risk of gastric cancer that may enter a surveillance and intervention program during the precancerous process is the most suitable strategy for decreasing mortality due to this malignancy. In addition, the determination of genotypes of $H$. pylori isolates in this population may allow us to further understand the relationship between putative virulence genes and clinical disease.

\section{Footnotes}

Authors' Contribution: All authors contributed to the revision of the manuscript, read, and approved the submitted version. Study design: Mohammad Kargar and Abbas Doosti. Literature review: Pouya Khodadadi and Mohammad Kargar. Data analysis: Pouya Khodadadi. Manuscript preparation: Pouya Khodadadi, Mohammad Kargar, Mahdi Bijanzadeh, Abbas Doosti, and Shapoor Aghaei.

Clinical Trial Registration Code: Cross sectional- Descriptive,IAUJB 47896213 
Conflict of Interests: The authors declare no conflict of interest.

Ethical Approval: The project and data collection were approved by the Ethics Committee of Islamic Azad University, Jahrom Branch (code: IAUJB 47896213).

Funding/Support: This research is part of a $\mathrm{PhD}$ thesis by Pouya Khodadadi, which was approved by the Jahrom Branch of Islamic Azad University, Iran.

\section{References}

1. Kargar M, Ghorbani-Dalini S, Doosti A, Najafi A. Five-year monitoring of considerable changes in tyrosine phosphorylation motifs of the Helicobacter pylori cagA gene in Iran. J Appl Genet. 2014;55(3):417-22. doi: 10.1007/s13353-014-0209-x. [PubMed: 24771298].

2. Watari J, Chen N, Amenta PS, Fukui H, Oshima T, Tomita T, et al. Helicobacter pylori associated chronic gastritis, clinical syndromes, precancerous lesions, and pathogenesis of gastric cancer development. World J Gastroenterol. 2014;20(18):5461-73. doi: 10.3748/wjg.v20.i18.5461. [PubMed: 24833876]. [PubMed Central: PMC4017061].

3. Kargar M, Souod N, Ghorbani-Dalini S, Doosti A. Evaluation of Helicobacter pylori isolates from gastric disorder patients in West of Iran. Sci Res Essays. 2011;6(31):6454-6458. doi: 10.5897/SRE11.826.

4. Talimkhani A, Mashak Z. Prevalence and Genotyping of Helicobacter pylori Isolated From Meat, Milk and Vegetable in Iran.JundishapurJMicrobiol.2017;10(11). doi: 10.5812/jjm.14240.

5. Pajavand H, Alvandi A, Mohajeri P, Bakhtyari S, Bashiri H, Kalali B, et al. High frequency of Helicobacter pylori isolates from patients with gastroduodenal disorders in Kermanshah, Iran. Jundishapur J Microbiol. 2015;8(11). e25425. doi: 10.5812/jjm.25425. [PubMed: 26862378]. [PubMed Central: PMC4740511].

6. Testerman TL, Morris J. Beyond the stomach: an updated view of Helicobacter pylori pathogenesis, diagnosis, and treatment. World J Gastroenterol. 2014;20(36):12781-808. doi: 10.3748/wjg.v20.i36.12781. [PubMed: 25278678]. [PubMed Central: PMC4177463].

7. Nakao M, Matsuo K, Ito H, Shitara K, Hosono S, Watanabe M, et al. ABO genotype and the risk of gastric cancer, atrophic gastritis, and Helicobacter pylori infection. Cancer Epidemiol Biomarkers Prev. 2011;20(8):1665-72. doi: 10.1158/1055-9965.EPI-11-0213. [PubMed: 21680535].

8. Liang L, Zhang Z, Bo W, Zhang M, Wang X. Association of different genotypes of Helicobacter pylori with CDX2 expression in intestinal metaplasia and gastric cancer. Int J Clin Experiment Med. 2019;12(9):11666-74.

9. Braga LL, Oliveira MA, Goncalves MH, Chaves FK, Benigno TG, Gomes $\mathrm{AD}$, et al. Helicobacter pylori strains of asymptomatic children from a high-risk gastric cancer area in northeastern Brazil. Mem Inst Oswaldo Cruz. 2014;109(8):1045-9. doi: 10.1590/0074-0276140279. [PubMed: 25494468]. [PubMed Central: PMC4325609].

10. Ghorbani-Dalini S, Kargar M, Doosti A, Najafi A. The relationship between Helicobacter pylori disease and bacterial count in stomach. Health. 2014;6(4):259-62. doi: 10.4236/health.2014.64038.

11. Jafari F, Shokrzadeh L, Dabiri H, Baghaei K, Yamaoka Y, Zojaji H, et al. vacA genotypes of cagA status and clinical outcomes in Iranian populations.Jpn Infect Dis. 2008;61(4):290-3. [PubMed: 18653971]. [PubMed Central: PMC3719049].

12. Aziz F, Chen X, Yang X, Yan Q. Prevalence and correlation with clinical diseases of $v a c A$ genotype among gastric patients from Northeast China. Biomed Res Int. 2014;2014:142980. doi: 10.1155/2014/142980. [PubMed: 24949419]. [PubMed Central: PMC4052682].

13. Ishaq S, Nunn L. Helicobacter pylori and gastric cancer: a state of the art review. Gastroenterol Hepatol Bed Bench. 2015;8(Suppl 1):S6-S14. [PubMed: 26171139]. [PubMed Central: PMC4495426].
14. Shiota S, Suzuki R, Yamaoka Y. The significance of virulence factors in Helicobacter pylori. J Dig Dis. 2013;14(7):341-9. doi: 10.1111/17512980.12054. [PubMed: 23452293]. [PubMed Central: PMC3721066].

15. Kalali B, Mejias-Luque R, Javaheri A, Gerhard M. H. pylori virulence factors: influence on immune system and pathology. Mediators Inflamm. 2014;2014:426309. doi: 10.1155/2014/426309. [PubMed: 24587595]. [PubMed Central: PMC3918698].

16. Wongphutorn P, Chomvarin C, Sripa B, Namwat W, Faksri K. Detection and genotyping of Helicobacter pylori in saliva versus stool samples from asymptomatic individuals in Northeastern Thailand reveals intra-host tissue-specific H. pylori subtypes. BMC Microbiol. 2018;18(1):10. doi: 10.1186/s12866-018-1150-7. [PubMed: 29378521]. [PubMed Central: PMC5789744].

17. Figueiredo C, Machado JC, Pharoah P, Seruca R, Sousa S, Carvalho R, et al. Helicobacter pylori and interleukin 1 genotyping: an opportunity to identify high-risk individuals for gastric carcinoma. J Natl Cancer Inst. 2002;94(22):1680-7. doi: 10.1093/jnci/94.22.1680. [PubMed: 12441323].

18. Saberi M, Momtaz H. Genotyping of cagA in Helicobacter pylori Strains in Saliva and Feces of Isfahan's Children. Navid No. 2018;20(64):24-33.

19. Yamaoka Y. Mechanisms of disease: Helicobacter pylori virulence factors. Nat Rev Gastroenterol Hepatol. 2010;7(11):629-41. doi: 10.1038/nrgastro.2010.154. [PubMed: 20938460].[PubMed Central: PMC3137895].

20. El Azeem EMA, Abdel-Ghaffar ARB, Shokaeir MH, Ali HA. Genotyping of Helicobacter pylori isolates from Egyptian Patients. Int J Biosci. 2017;10(4):121-8. doi: 10.12692/ijb/10.4.121-128.

21. Torres LE, Melian K, Moreno A, Alonso J, Sabatier CA, Hernandez $\mathrm{M}$, et al. Prevalence of Helicobacter pylori isolates. World J Gastroenterol. 2009;15(2):204-10. doi: 10.3748/wjg.15.204. [PubMed: 19132771]. [PubMed Central: PMC2653313].

22. Dabiri H, Jafari F, Baghaei K, Shokrzadeh L, Abdi S, Pourhoseingholi $\mathrm{MA}$, et al. Prevalence of $b a b B$ genotypes in Iranian dyspeptic patients. Microb Pathog. 2017;105:226-30. doi: 10.1016/j.micpath.2017.02.018. [PubMed: 28215588].

23. Alaoui Boukhris S, Amarti A, El Rhazi K, El Khadir M, Benajah DA, Ibrahimi SA, et al. Helicobacter pylori genotypes associated with gastric histo-pathological damages in a Moroccan population. PLoS One. 2013;8(12). e82646. doi: 10.1371/journal.pone.0082646. [PubMed: 24349327]. [PubMed Central: PMC3857243].

24. Foegeding NJ, Caston RR, McClain MS, Ohi MD, Cover TL. An Overview of Helicobacter pylori VacA Toxin Biology. Toxins (Basel). 2016;8(6). doi: 10.3390/toxins8060173. [PubMed: 27271669]. [PubMed Central: PMC4926140].

25. Wen S, Moss SF. Helicobacter pylori virulence factors in gastric carcinogenesis. Cancer Lett. 2009;282(1):1-8. doi: 10.1016/j.canlet.2008.11.016. [PubMed: 19111390]. [PubMed Central: PMC2746929].

26. Takahashi-Kanemitsu A, Knight CT, Hatakeyama M. Molecular anatomy and pathogenic actions of Helicobacter pylori CagA that underpin gastric carcinogenesis. Cell Mol Immunol. 2020;17(1):50-63. doi: 10.1038/s41423-019-0339-5. [PubMed: 31804619]. [PubMed Central: PMC6952403].

27. Hosseini E, Poursina F, de Wiele TV, Safaei HG, Adibi P. Helicobacter pylori in Iran: A systematic review on the association of genotypes and gastroduodenal diseases. J Res Med Sci. 2012;17(3):280-92. [PubMed: 23267382]. [PubMed Central: PMC3527048].

28. Soleimani N, Mohabati Mobarez A, Farhangi B. Cloning, expression and purification flagellar sheath adhesion of Escherichia coli host as a vaccination target. Clin Exp Vaccine Res. 2016;5(1):19-25. doi: 10.7774/cevr.2016.5.1.19. [PubMed: 26866020]. [PubMed Central: PMC4742594].

29. Farshad S, Japoni A, Alborzi A, Hosseini M. Restriction fragment length polymorphism of virulence genes Helicobacter pylori strains isolated from Iranian patients with gastric ulcer and nonulcer disease. Saudi Med J. 2007;28(4):529-34. [PubMed: 17457472]. 
30. Souod N, Kargar M, Doosti A, Ranjbar R, Sarshar M. Genetic Analysis of Helicobacter Pylori Isolates and Their Relationship with Gastroduodenal Diseases in the West of Iran. Iran Red Crescent Med J. 2013;15(5):371-5. doi: 10.5812/ircmj.3732. [PubMed: 24349721]. [PubMed Central: PMC3838643].

31. Kamogawa-Schifter Y, Yamaoka Y, Uchida T, Beer A, Tribl B, SchonigerHekele M, et al. Prevalence of Helicobacter pylori and its CagA subtypes in gastric cancer and duodenal ulcer at an Austrian tertiary referral center over 25 years. PLoS One. 2018;13(5). e0197695. doi 10.1371/journal.pone.0197695. [PubMed: 29813089]. [PubMed Central: PMC5973618].

32. Bahadori A, Somi MH, Doran F, Eftekharsadat AT. Determination of correlation between principal genotypes of vacA genotypes and clinical out come in patients suffering from active chronic gastritis and gastric adenocarcinoma from Iran and Turkey. Biomed Res Int. 2017;28(4):1743-8.

33. Roman-Roman A, Martinez-Carrillo DN, Atrisco-Morales J, AzucarHeziquio JC, Cuevas-Caballero AS, Castanon-Sanchez CA, et al. babA2 increase the risk of ulcer and gastric cancer in patients from Southern Mexico. Gut Pathog. 2017;9:18. doi: 10.1186/s13099-017-0167-z. [PubMed: 28413454]. [PubMed Central: PMC5390388].
34. Pinto-Ribeiro I, Ferreira RM, Batalha S, Hlaing T, Wong SI, Carneiro F, et al. Helicobacter pylori vacA Genotypes in Chronic Gastritis and Gastric Carcinoma Patients from Macau, China. Toxins (Basel). 2016;8(5). doi: 10.3390/toxins8050142. [PubMed: 27164143]. [PubMed Central: PMC4885057].

35. Yamaoka Y, Kato M, Asaka M. Geographic differences in gastric cancer incidence can be explained by differences between Helicobacter pylori strains. Intern Med. 2008;47(12):1077-83. doi: 10.2169/internalmedicine.47.0975. [PubMed: 18552463]. [PubMed Central: PMC3732488].

36. Yamaoka Y, Orito E, Mizokami M, Gutierrez O, Saitou N, Kodama T, et al. Helicobacter pylori in north and South America before Columbus. FEBS Lett. 2002;517(1-3):180-4. doi: 10.1016/S0014-5793(02)02617-0.

37. Dabiri $H$, Bolfion M, Mirsalehian A, Rezadehbashi M, Jafari F, Shokrzadeh L, et al. Analysis of Helicobacter pylori genotypes in Afghani and Iranian isolates. Pol J Microbiol.2010;59(1):61-6. [PubMed: 20568532]. [PubMed Central: PMC3126918].

38. Amer FA. Helicobacter pylori genotypes among patients in a university hospital in Egypt: identifying the determinants of disease severity. J Microbiol Infect Dis. 2013;3(3):109-15. doi: 10.5799/ahinjs.02.2013.03.0092. 